This item was submitted to Loughborough's Research Repository by the author.

Items in Figshare are protected by copyright, with all rights reserved, unless otherwise indicated.

\title{
Polymer electrolyte membrane fuel cell fault diagnosis and sensor abnormality identification using sensor selection method
}

\section{PLEASE CITE THE PUBLISHED VERSION}

https://doi.org/10.1016/j.jpowsour.2019.227394

\section{PUBLISHER}

Elsevier

VERSION

AM (Accepted Manuscript)

\section{PUBLISHER STATEMENT}

This paper was accepted for publication in the journal Journal of Power Sources and the definitive published version is available at https://doi.org/10.1016/j.jpowsour.2019.227394.

LICENCE

CC BY-NC-ND 4.0

\section{REPOSITORY RECORD}

Mao, Lei, Lisa Jackson, Weiguo Huang, Zhinong Li, and B Davies. 2019. "Polymer Electrolyte Membrane Fuel Cell Fault Diagnosis and Sensor Abnormality Identification Using Sensor Selection Method". Loughborough University. https://hdl.handle.net/2134/14052377.v1. 


\title{
.Polymer Electrolyte Membrane Fuel Cell Fault Diagnosis and Sensor Abnormality
} Identification using Sensor Selection Method \\ ${ }^{1}$ Department of Precision Machinery and Precision Instrumentation, University of Science \\ and Technology of China, Hefei, China
}

${ }^{2}$ Key Laboratory of Precision Scientific Instrumentation of Anhui Higher Education Institutes, University of Science and Technology of China, Hefei, China

${ }^{3}$ Department of Aeronautical and Automotive Engineering, Loughborough University, Loughborough, UK

${ }^{4}$ School of Rail Transportation, Soochow University, Suzhou, China

${ }^{5}$ Key Laboratory of Nondestructive Testing, Ministry of Education, Nanchang HangKong University, Nanchang, China, 330063

Corresponding Author: 1eimao82@ustc.edu.cn

\begin{abstract}
:
In this study, a sensor selection technique is proposed to identify various system faults including polymer electrolyte membrane (PEM) fuel cell stack faults and faults of ancillary systems, thus improving system reliability and durability. With proposed technique, the information in sensors could be investigated without fuel cell numerical model, and sensors more sensitive to the fuel cell system performance change could be identified for reliable fault diagnosis. Moreover, the reliability of sensors can be evaluated during the system operation with proposed technique. The performance of selected sensors with proposed technique in identifying fuel cell system faults is investigated using test data from a PEM fuel
\end{abstract}


cell system, where the data-driven fault diagnostic framework is applied. Results demonstrate that with the selected sensors, different levels of fuel cell stack faults can be distinguished with good quality, and the sensor faults can also be identified during the fuel cell operation. Therefore, the proposed sensor selection technique can be beneficial in practical PEM fuel cell systems for the identification of various system faults, from which mitigation strategies could be taken to improve the system reliability and durability, while the maintenance cost could be reduced by avoiding unnecessary system stop and maintenance actions.

Key word: polymer electrolyte membrane fuel cell, fault diagnosis, data-based approach, sensor selection, fuel cell flooding, sensor fault

\section{Introduction}

As an alternative energy source, hydrogen and fuel cell system, especially PEM fuel cell, has attracted much more attention in the last few decades due to the characteristics like zero emission and high efficiency, compared to other energies sources such as lithium-ion battery, photovoltaic. On this basis, a series of practical applications utilizing PEM fuel cell technologies have emerged, such as stationary power station, consumer device, automotive.

However, limited reliability and durability are still two major obstacles preventing PEM fuel cell system from further commercialization. To address these issues, a set of studies have been devoted to the PEM fuel cell system fault diagnosis and prognosis, from which the system state and its remaining useful life (RUL) could be evaluated. With fault diagnostic techniques, PEM fuel cell faults could be identified, and mitigation strategies could be taken to remove the faults; while from PEM fuel cell prognosis, the future performance could be predicted for the design of maintenance actions, where the system useful life could be extended. 
In PEM fuel cell fault diagnosis, either model-based or data-driven techniques could be applied to identify the faults. With model-based techniques, PEM fuel cell model should be developed, and the faults could be identified by analyzing the residuals between model outputs and actual measurements ${ }^{[1]}$. Forrai et al. ${ }^{[2]}$ used equivalent circuit model to simulate the PEM fuel cell voltage and internal impedance, the model parameters were determined using a set of characterization tests. With configured model, PEM fuel cell performance decay could then be investigated by analyzing variation of model parameters at different operating conditions. In ${ }^{[3]}$, Fouquet et al. proposed a modified Randles model to represent the PEM fuel cell internal impedance. With impedance measurements, the model parameters sensitive to the membrane water content could be studied, thus fuel cell flooding and drying out could be identified by monitoring the model parameter change. Ingimundarson et al. ${ }^{[4]}$ proposed mass balance equations taking into account of natural leak of stack and humidity, to investigate the detection of hydrogen leaks in the PEM fuel cell stack. Rubio et al. ${ }^{[5]}$ investigated the discrimination of various PEM fuel cell faults, including flooding, drying out, and catalyst poisoning, using equivalent circuit model. Two models were used in the study, one was used for diagnosis of flooding and drying out scenarios, the other was used for the detection of catalyst poisoning. For reliable fault diagnosis, the parameters from two models should be determined by performing a set of tests. In $^{[6]}$, a radial basis function network was proposed for the intelligent fault detection in PEM fuel cell systems, model inputs included stack current and compressor motor voltage, while outputs from the model were net power, oxygen excess ratio, and stack voltage. With proposed model, different sensor faults could be identified. Similarly, Mohammadi et al. ${ }^{[7]}$ used neural network to simulate PEM fuel cell behaviour, where effects of temperature, pressure, and humidity on equivalent circuit model parameters like resistance and capacitor were investigated. From above studies, it can be concluded that most model-based fault diagnostic studies use equivalent circuit model or 
black box like neural networks for simulating PEM fuel cell behaviour, thus extra tests should be performed to determine model parameters or provide sufficient data for neural network training, which is time-consuming and would interrupt the normal PEM fuel cell system operation.

With data-driven diagnostic techniques, PEM fuel cell model is not required, and data from PEM fuel cell system is analysed for the fault identification ${ }^{[8]}$. Andujar et al. ${ }^{[9]}$ provided a review of methodologies for PEM fuel cell fault detection and performance improvement, where states of various components in the system, including electrical state, thermal state, fuel state, cell state, and stack repair and improvement state, were analysed with fault diagnostic techniques. Lin et al. ${ }^{[10]}$ presented a review about hydrogen fuel cell condition monitoring and prediction methods, results showed that data-based and filter-based techniques were more widely used for fuel cell fault diagnosis and prognosis. Steiner et al. ${ }^{[1]}$ proposed a method of identifying PEM fuel cell flooding using cell voltages, where wavelet packet transform was applied to obtain signal energies at different frequency ranges. Li et al. ${ }^{[12]}$ analysed individual cell voltages from a PEM fuel cell stack using fisher discriminant analysis (FDA) and directed acyclic graph support vector machine (DAGSVM). From the results different PEM fuel cell faults, including high current pulse, cooling water stop, high/low air stoichiometry, and CO poisoning, could be identified. Kim et al. ${ }^{[13]}$ analysed the frequency components in the PEM fuel cell voltage at different hydrogen and air supply rates, from which relationship between root mean square frequency (RMSF), autocorrelation standard deviation (ACSD) and hydrogen/air supply rates were determined for PEM fuel cell state monitoring. Liu et al. ${ }^{[14]}$ proposed a PEM fuel cell diagnostic method using extreme learning machine and Dempster-Shafer evidence theory, where multiple measurements such as hydrogen and air flow rate, pressure, temperature were used in the diagnosis. Placca et al. ${ }^{[15]}$ applied principal component analysis (PCA) to explore correlations between sensor 
measurements and PEM fuel cell stack behaviour, where a set of sensor measurements such as temperature, pressure, voltage, relative humidity were included in the analysis. Pahon et al. ${ }^{[16]}$ used wavelet transform to decompose signals from different sensors (stack voltage, individual cell voltages, and single phase air pressure drop), and the most energetic detail change was used for identification of PEM fuel cell faults due to high air stoichiometry. Liu et al. ${ }^{[17]}$ used discrete hidden Markov model (DHMM) in PEM fuel cell system state evaluation in tramways, results demonstrated that the proposed method could perform diagnosis with less computation cost and high accuracy. Mao et al. ${ }^{[18]}$ proposed a PEM fuel cell fault diagnostic framework using a set of sensor measurements, where kernel PCA was applied for dimension reduction of raw dataset, wavelet packet transform was used for feature extraction, singular value decomposition was selected for feature selection. From results several PEM fuel cell faults, including membrane drying out and hydrogen issue, could be identified.

Furthermore, several studies have been devoted to the PEM fuel cell prognosis. Adaptive neuron fuzzy inference system (ANFIS) has been widely applied due to its advantages from neural network and fuzzy logic technique ${ }^{[19-20]}$. With proper definitions of model inputs and outputs, the trained ANFIS model could provide reasonable prediction for PEM fuel cell performance. Moreover, other techniques, including filtering approaches ${ }^{[21]}$, recurrent neural network ${ }^{[22]}$, and deep learning method ${ }^{[23]}$, have also been applied for PEM fuel cell performance prediction, where PEM fuel cell RUL can be evaluated. Besides above modelbased techniques, the fuzzy logic related techniques have also been applied for PEM fuel cell prognosis, where the fuzzy rules could be generated using expert knowledge or test data, with which the inference could then be performed to predict the system future performance. Kishor and Mohanty ${ }^{[24]}$ used fuzzy technique to predict several PEM fuel cell system parameters, like cathode pressure, stack voltage, oxygen and hydrogen partial pressures, 
while Li et al. ${ }^{[25-26]}$ generated fuzzy rules for power management of the fuel cell/battery/super-capacitor hybrid tramways, from which multiple power sources could be coordinated to avoid power demand rapid change.

It can be found from above studies that the key for the reliable PEM fuel cell fault diagnosis and prognosis is the collection and utilization of sufficient information from the PEM fuel cell system. According to previous results ${ }^{[27]}$, various PEM fuel cell failure mechanisms could cause variations in different sensors, thus the use of multiple sensors could provide more distinguishable information for PEM fuel cell faults ${ }^{[28-29]}$. For this purpose, various sensors, such as current sensor, voltage sensor, thermocouple, flow meter, pressure gauge, humidification sensor, are installed in PEM fuel cell system to collect the system information, leading to a high dimensional dataset during the system operation.

As the analysis of high dimensional dataset is complex and time-consuming, the direct analysis cannot meet the on-line monitoring requirement. Moreover, as different PEM fuel cell failure mechanisms have different influences in different sensors ${ }^{[30]}$, sensors would show different sensitivities to these failure scenarios, and inclusion of insensitive sensors may not improve the diagnostic results but only increases the computational time. Therefore, it is necessary to further investigate the information in the sensors, and select sensors which are more sensitive to PEM fuel cell system performance variation in fault diagnosis.

For this purpose, sensor selection algorithms have been proposed and applied in PEM fuel cell system to determine the optimal sensors for reliable fault diagnosis, and the cost of monitoring system can also be reduced significantly by avoiding the use of expensive sensors. Several researchers have performed analysis to investigate the effects of various PEM fuel cell parameters on its performance, such as cell temperature ${ }^{[31-32]}$, anode and cathode pressure $^{[33]}$, stoichiometry and relative humidity ${ }^{[34]}$, from which the sensitivities of sensors to 
PEM fuel cell performance decay can be clarified. Moreover, PEM fuel cell physical model, which is the so-called "white-box" model and include sets of differential equations describing mass and momentum conservation inside the PEM fuel cell, can be developed to simulate the PEM fuel cell behaviour, where sensor sensitivities to different fuel cell faults can be calculated. From the results, several sensor selection methods have been proposed to determine optimal sensors using different selection criteria, including sensor resistance to the measurement noise ${ }^{[35]}$, and sensor capability in identifying different PEM fuel cell failure modes ${ }^{[36]}$. Results demonstrate that with selected sensors, reliable PEM fuel cell fault diagnosis can be obtained with reasonable computational time ${ }^{[37]}$. Recently, a technique was proposed to identify sensor faults in fuel cell system ${ }^{[38]}$, where TS fuzzy model is used to simulate fuel cell system, and unknown input observer and fault estimator were calculated using linear matrix equalities and inequalities.

However, due to the multi-domain knowledge included in the PEM fuel cell model development, the development of accurate PEM fuel cell model including all the failure mechanisms is extremely difficult, and some failure mechanisms, such as influence of liquid water in blocking gas channel, could not be modelled accurately. Therefore, in the previous studies, only limited PEM fuel cell faults are considered in the sensor selection analysis ${ }^{[35-36]}$. Moreover, due to the assumptions used in the PEM fuel cell model development, like constant relative humidity throughout the PEM fuel cell, some internal behaviour including humidity distribution inside the fuel cell cannot be simulated, thus corresponding sensor measurements such as relative humidity cannot be analysed with the developed model. Therefore, with developed PEM fuel cell model, only limited PEM fuel cell faults and sensor measurements can be analysed, which restricts the application of sensor selection algorithms in practical PEM fuel cell systems, where more PEM fuel cell faults and sensors should be considered. 
Furthermore, when sensors are used for fuel cell fault diagnosis, it is always assumed that the operation has not been investigated in previous studies. However, in practical applications, the reliability of sensors during the system operation may change, and the inclusion of inaccurate sensor measurements will provide misleading results in both PEM fuel cell fault diagnosis and prognosis, since the measurements of abnormal sensors do not represent the actual state of PEM fuel cell system ${ }^{[39]}$. Therefore, it is necessary to determine the optimal sensors with consideration of sensor reliability, thus sensors providing abnormal measurements could be identified and removed from fault diagnosis and prognosis.

In this study, an efficient sensor selection algorithm is proposed to select sensors for fault diagnosis, and the performance of selected sensors in distinguishing different PEM fuel cell system faults is investigated. The major contribution of this work is that the proposed sensor selection technique can select the sensors without the PEM fuel cell physical model, this can largely reduce complexity and computational effort of the analysis, since the development of PEM fuel cell physical model is difficult and time-consuming. Moreover, the sensor reliability during the fuel cell operation can be investigated with the proposed technique, and abnormal sensors, i.e. sensors providing inaccurate measurements and not representing the actual fuel cell physical properties, can be identified effectively. Therefore, the proposed technique can identify various PEM fuel cell system faults, including PEM fuel cell faults (flooding in the study) and faults of ancillary systems (sensor faults in the study). From the results, fuel cell faults could be identified at their early stage, thus mitigation strategies could be taken to remove the fault effects and recover the system performance, and fuel cell system useful life could be extended; while by identifying abnormal sensors with proposed technique, the unnecessary system stop and maintenance could be avoided to save the financial cost, while system reliability could be improved. In section 2 , the proposed sensor selection 
algorithm is described, and its use in identifying abnormal sensors is also presented. Section 3 describes the tested PEM fuel cell system and test data at different system states. In section 4, the optimal sensors are selected with proposed technique. In section 5, the performance of selected sensors in distinguishing fuel cell fault is investigated using data-driven fault diagnostic approaches including kernel principal component analysis (KPCA), wavelet packet transform (WPT), singular value decomposition (SVD). Furthermore, the capability of proposed techniques in identifying abnormal sensors is also investigated in section 5 . From the results, conclusions are given in section 6 .

\section{Proposed sensor selection technique}

As described in section 1, the proposed sensor selection technique does not require PEM fuel cell physical model, and could evaluate sensor sensitivity with the test data collected from the PEM fuel cell system. In this study, a set of sensors installed at different locations of PEM fuel cell system are used for the analysis, including voltage sensor, thermocouple, flow meter, pressure sensor, and humidification sensor at anode and cathode sides, this will be further described in section 3 .

In the analysis, each sensor measurement within certain operation period is analysed using wavelet packet transform (WPT), which decomposes the original signal into wavelet packets at different frequency ranges, signals at these frequency ranges are then re-constructed for calculating signal energies. From the results, the two highest energies are selected to evaluate the sensor state, this will be further illustrated in section 4. The reason of selecting WPT is that wavelet transform-based techniques have been widely used in many applications for feature extraction and condition evaluation purpose ${ }^{[8,16]}$. Compared to wavelet transform (WT), WPT can decompose the original signal at both high and low frequency ranges, thus can extract more wavelet coefficients. 
In order to study the sensor consistency during the operation, the same analysis described above is applied to the sensor measurements collected at two consecutive periods. From the results, two sets of energy features can be generated for each sensor, from which the Euclidean distance can be calculated. The consistency of a sensor to the PEM fuel cell performance can then be expressed using Eq.(1), which is the ratio between the sensor state and PEM fuel cell performance represented with the fuel cell voltage. It should be noted that in the current study, it is assumed that the fuel cell voltage can always be measured accurately, thus the voltage variation is due to the change of fuel cell system state.

$$
\mathrm{CONi}=\mathrm{EDi} / \mathrm{ED}_{\mathrm{v}}
$$

where $\mathrm{CONi}$ is the sensor consistency to the PEM fuel cell performance, EDi is the Euclidean distance calculated using features from the $i$ th sensor, and EDv is the Euclidean distance calculated using features from the fuel cell voltage measurements.

It should be mentioned that the Euclidean distance between sensor features at two consecutive periods ( $t_{1}$ and $t_{2}$ for instance) is expressed below.

$$
\mathrm{ED}=\sqrt{\left(x_{2}-x_{1}\right)^{2}+\left(y_{2}-y_{1}\right)^{2}}
$$

Where $x_{1}$ and $y_{1}$ are the two features extracted using WPT from corresponding sensor measurement at $t_{1}$, while $x_{2}$ and $y_{2}$ are the two features extracted using WPT from corresponding sensor measurement at $t_{2}$.

With Eq. (1), the sensor consistency to PEM fuel cell system can be calculated. It is expected that for a sensor more sensitive to fuel cell performance change, its measurement will show larger variation. Therefore, the sensor more sensitive to the fuel cell performance variation can be determined using the ratio between the sensor state variation and the PEM fuel cell state change, which can be expressed with Eq. (3). 
where $\mathrm{CA}_{\mathrm{i}}$ is the $i$ th sensor sensitivity to the PEM fuel cell performance change, and $\mathrm{CON} \mathrm{i}$ is the sensor consistency calculated with Eq. (1), using sensor measurements at two periods with both representing fuel cell normal state, which is defined herein as the condition without fuel cell performance decay, i.e. without the drop of fuel cell voltage; while CONif is the sensor consistency calculated using sensor measurements at two periods with one representing fuel cell normal state, and the other representing fuel cell faulty state, i.e. with the drop of fuel cell voltage.

With proposed technique, the sensor sensitivity can be evaluated. Sensor with $C A$ value of larger than 1indicates its higher sensitivity to the PEM fuel cell performance change, thus should have higher probability to be selected, while sensor with $C A$ value of smaller than 1 means it is less sensitive to the PEM fuel cell performance change, and should not be included in the PEM fuel cell fault diagnosis. This will be further illustrated in section 4.

As described before, the sensor reliability can also be investigated using proposed method, this could be performed by investigating the evolution of sensor consistency using Eq.(1). In cases where voltage drop is not observed, the sudden and significant variation in the sensor consistency indicates the reliability loss of corresponding sensor. From the results, the abnormal sensors can be excluded from the selected sensors for reliable fault diagnosis. This will be further illustrated in section 5.2 .

It can be summarized that as fewer sensors which are more sensitive to fuel cell faults are used in the analysis, the reliable diagnostic results can be obtained with less computation time. Moreover, by identifying and removing abnormal sensors, the accurate diagnostic performance can be provided. It should be highlighted that since PEM fuel cell physical model is not required, the proposed technique can determine the sensors for fault diagnosis 
using data collected from the fuel cell, thus can be better applied in practical applications, where a set of sensors are installed and can provide continuous measurements during the PEM fuel cell operation.

\section{Description of tested PEM fuel cell system}

A PEM fuel cell system with capability of $80 \mathrm{~W}$ is used in the test, which is depicted in Figure 1(a), including a single-cell, cooling system, and ancillary systems supplying air and hydrogen. The PEM fuel cell is water cooled using deionised water sent through a serpentine channel in between cells in the cell. The gases are humidified through Nafion tube, bubbler type humidifiers, and are specified to humidify the feed gases at the range of flow rates to be used. Temperature is controlled both at the bottle and the heated length of the tube.

As single cell is used in the test, enough heat cannot be produced for PEM fuel cell operation at the required temperature, thus a cell heating strategy is used, where the coolant water is heated to the desired temperature for the cell, and then pumped though the cell to produce enough heat.

It should be noted that an electronic load bank with the range of $0-150 \mathrm{~A}$ and $0-800 \mathrm{~W}$ is used in the test to consume the energy developed by the cell and log/display the energy use in real time.
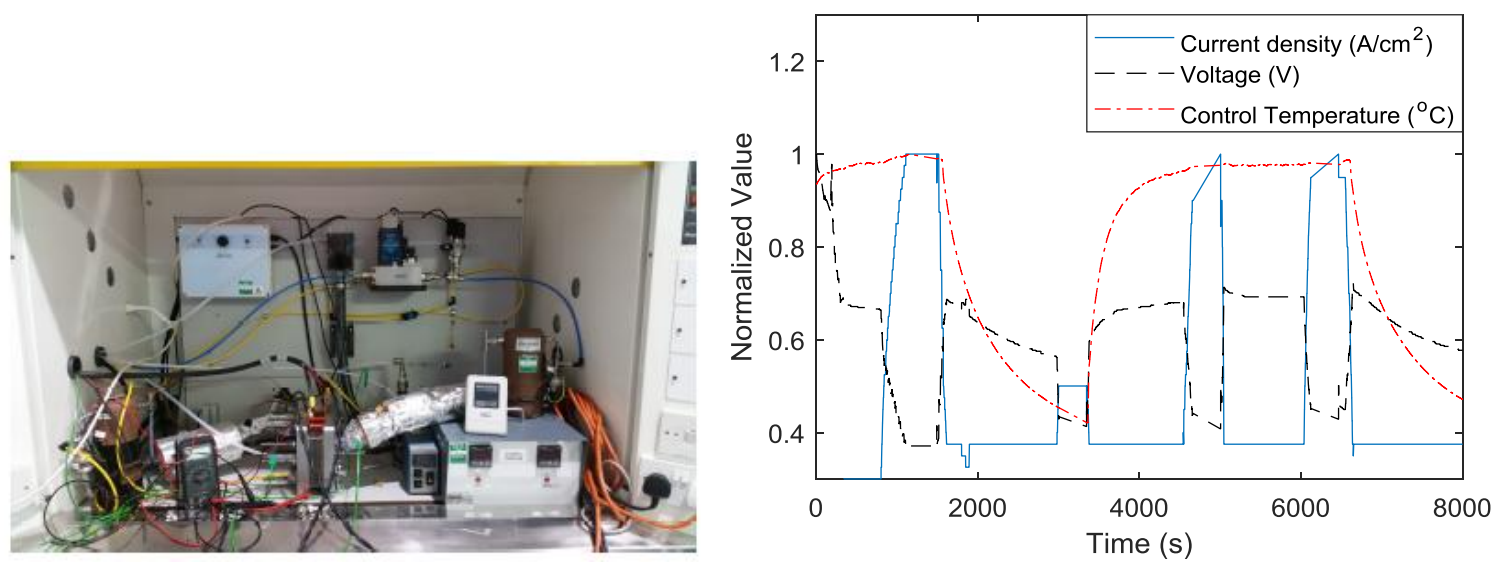
The tested PEM fuel cell has active area of $100 \mathrm{~cm}^{2}$ and is manufactured by Pragma Industries using the same materials and technologies as commercial PEM fuel cells, including Nafion polymer electrolyte membrane, platinum nano-particle catalyst, carbon diffusion materials, silicone sealing gaskets, and graphite flow field plates. In the test, a set of sensors, including flow meter, humidification sensor, pressure sensor, and thermocouple are installed at anode and cathode outlets to collect the information during the fuel cell operation, these sensors are used as in practical PEM fuel cell systems to collect sufficient information from the system and in the following analysis for the PEM fuel cell fault diagnosis, which are listed in Table 1. It should be mentioned that several sensors are also installed at anode and cathode inlets for information collection, such as anode and cathode inlet flow meters, anode and cathode thermocouple, anode and cathode pressure gauges, which are used to control the operating condition of PEM fuel cell system and not used in the fault diagnosis, thus these sensors are not listed in Table 1. Table 2 lists the technical details of the membrane electrode assembly (MEA) in PEM fuel cell.

Table 1 Sensors used in the fuel cell test and fault diagnosis

\begin{tabular}{|c|c|c|c|}
\hline No. & Sensor /Symbol & No. & Sensor /Symbol \\
\hline 1 & Cathode outlet flow meters $/ \mathrm{W}_{\mathrm{ca}}$ & 7 & Stack thermocouple/T \\
\hline 2 & Anode outlet flow meter $/ \mathrm{W}_{\mathrm{an}}$ & 8 & Cathode outlet thermocouple/ $\mathrm{T}_{\mathrm{ca}}$ \\
\hline 3 & $\begin{array}{c}\text { Cathode outlet humidification } \\
\text { sensor/ } \mathrm{RH}_{\mathrm{ca}}\end{array}$ & 9 & Anode outlet thermocouple/ $\mathrm{T}_{\mathrm{an}}$ \\
\hline 4 & $\begin{array}{c}\text { Anode outlet humidification } \\
\text { sensor } / \mathrm{RH}_{\mathrm{an}}\end{array}$ & 10 & Current/I \\
\hline 5 & Cathode outlet pressure gauge $/ \mathrm{P}_{\mathrm{ca}}$ & 11 & Voltage/V \\
\hline
\end{tabular}




\begin{tabular}{cc}
\hline Parameter & Value \\
\hline Membrane thickness $(\mu \mathrm{m})$ & 25 \\
Active area $\left(\mathrm{cm}^{2}\right)$ & 100 \\
Platinum loading $\left(\mathrm{mg} / \mathrm{cm}^{2}\right)$ & 0.2 \\
Gas diffusion thickness $(\mu \mathrm{m})$ & 415 \\
\hline
\end{tabular}

During the PEM fuel cell system operation, the fuel cell flooding is activated by reducing the PEM fuel cell temperature, this could facilitate the generation and accumulation of liquid water inside the cell to block gas path to the catalyst layer, and finally cause PEM fuel cell performance decay. With the observation of clearly PEM fuel cell voltage drop in the test, the temperature is then increased to remove the liquid water and recover the fuel cell performance. Based on previous studies ${ }^{[8]}$, the fuel cell flooding is caused by accumulation of liquid water inside the fuel cell, and can lead to the largest performance degradation rate, while with proper mitigation strategies, the flooding effect can be effectively removed without causing permanent damage to the fuel cell, which is consistent with the observations in the test. This process is repeated several times in the test to confirm its repeatability. Figure 1(b) shows the variation of current density and PEM fuel cell voltage due to the control temperature change, where the control temperature is the cell temperature $(\mathrm{T})$ in Table 1 , since the temperature is controlled herein to generate flooding scenario. It should be mentioned that these measurements are normalized for better illustration purpose. It can be seen from Figure 1(b) that the fuel cell voltage change is only due to the control temperature, as current density at this time period is kept constant. The current density change at period 
about $1000-1500 \mathrm{~s}, 3000-3400 \mathrm{~s}, 4500-5000 \mathrm{~s}$, and $6000-6500 \mathrm{~s}$ is due to the fact that

polarization curve is collected from the PEM fuel cell system to evaluate PEM fuel cell state, which leads to the lower voltage during these period. The polarization curves collected from PEM fuel cell will not be analysed in the current study. It should be mentioned that the reason of using cell temperature reduction for flooding scenario is that the load current would not be changed during this process, thus the discrimination of normal operation and flooding could be performed at same current density.

Moreover, it is noted that only the test data at the $1^{\text {st }}$ cycle (between about 1500 s and 3000 s) is analysed in sections 4 and 5.1 for fault diagnosis of PEM fuel cell faults (flooding herein), since some sensor measurements after 3000s become unreliable, which will be further studied in section 5.2.

\section{Selection of optimal sensors with proposed technique}

As described in section 2, with Eqs.(1) and (2), sensor measurements collected at different operation periods can be used to determine the sensor sensitivity to the fuel cell system performance change. In the study, sensor measurements at PEM fuel cell normal condition and flooding scenario are used to determine the optimal sensors for fault diagnosis. It should be noted that the PEM fuel cell flooding is defined with the voltage drop exceeding 5\%, thus capability of proposed approach in identifying the early stage PEM fuel cell fault can be investigated, as the early stage flooding can be removed with appropriate mitigation strategies. It should be noted that as the voltage drop is caused by reducing the PEM fuel cell temperature, so that cell temperature is lower than cathode gas dew point temperature, leading to water condensation at cathode side, moreover, by increasing the cell temperature after the voltage drop, the voltage can be recovered to its normal value (as shown in Figure 1b). Therefore, it can be confirmed that the voltage drop in the test is due to the PEM fuel cell 
flooding, since flooding is caused by generation and accumulation of liquid water inside the PEM fuel cell, which can be induced by PEM fuel cell temperature reduction, and flooding can cause reversible PEM fuel cell degradation, i.e. the degradation due to flooding can be recovered efficiently using proper mitigations like increasing PEM fuel cell temperature ${ }^{[8]}$.

As described in section 2, since both low pass results (approximation) and high pass results (detail) could be obtained using WPT by letting original data passing through filters, more wavelet packets can be extracted at various frequency ranges. With WPT applied to each sensor measurement, wavelet packets from different frequency ranges can be extracted with Eq. (4).

$$
C_{j, k}=\int f(t) \psi_{j, k}(t) d t
$$

Where $f(t)$ is the original signal, $\Psi_{j, k}$ is the wavelet function, $j$ and $k$ are the scale and shift parameters determining the wavelet function shape.

With extracted wavelet packets, the signal at different frequency ranges can be re-constructed, from which signal energies can be calculated and used to evaluate their contributions to the original sensor measurement.

Eq. (5) expresses the re-constructed signal at jth decomposition level, and energy of reconstructed signal can be calculated using Eq. (6).

$$
f_{j}(t)=C \sum_{k} C_{j, k} \boldsymbol{\Psi}_{j, k}(t)
$$

where $\mathrm{C}$ is a constant independent of signals.

$$
E_{s}=\int\left|f_{j}(t) f_{j}(t)\right| d t
$$


From the signal energies at different frequency ranges, the two highest energies are selected in the study as features (such as $x_{1}$ and $y_{1}$ in Eq. 2) to represent the sensor state.

With the proposed approach and collected measurements from sensors listed in Table 1, sensor consistency to the PEM fuel cell performance change can be obtained using Eq.(3), the results are depicted in Figure 2. It should be noted that the symbols in horizontal axis of Figure 2 represent various sensors used in the test, their physical meanings can be found in Table 1.

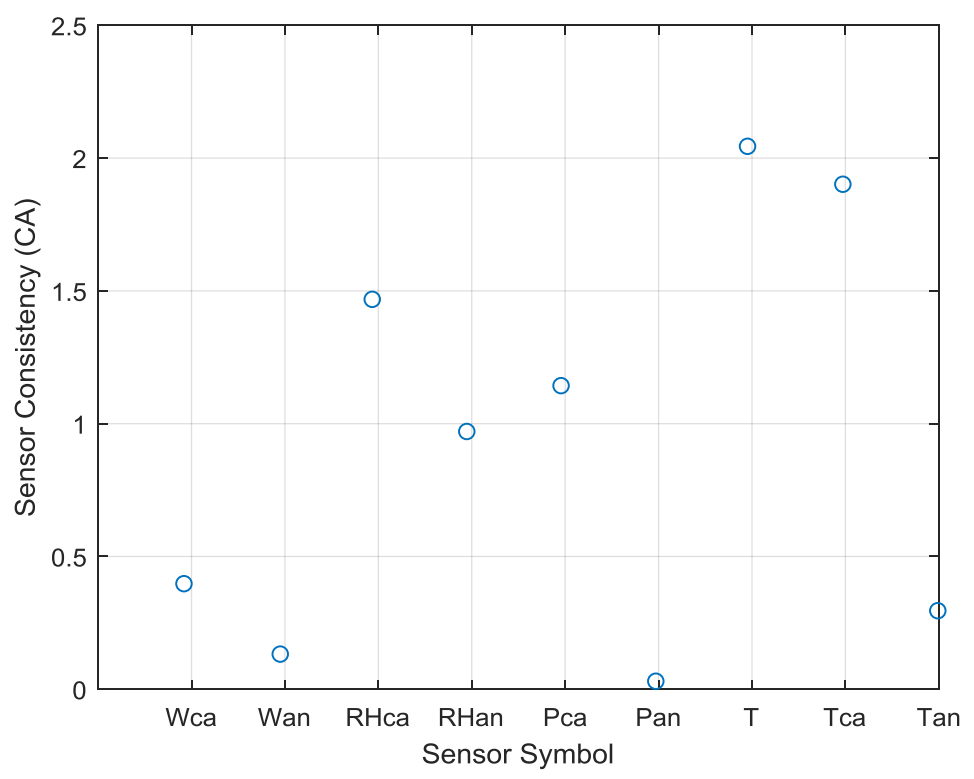

Figure 2 Distribution of sensor sensitivities

It can be seen from Figure 2 that five sensors, including T, $\mathrm{T}_{\mathrm{ca}}, \mathrm{RH}_{\mathrm{ca}}, \mathrm{P}_{\mathrm{ca}}$, and $\mathrm{RH}$ an, have the sensitivity values around or higher than 1 , indicating these sensors are more sensitive to the fuel cell performance variation, thus should be selected in PEM fuel cell fault diagnosis. Moreover, as fuel cell flooding is caused by reducing cell temperature and accumulation of liquid water accumulation at cathode side, $\mathrm{T}$ and Tca would be affected significantly in this process, while Tan would not show clearly variation as no liquid water accumulation at anode in the test. As gas dew points at anode and cathode sides are set to different values in the test, 
only the water condensation at cathode side is induced in the test when the cell temperature is lower than the gas dew point temperature at cathode side, thus water condensation and cell flooding is not sensitive to Tan in the study.

By comparing with sensors selected from previous selection algorithms ${ }^{[32-34]}$, it is found that the same sensors including $\mathrm{T}, \mathrm{T}_{\mathrm{ca}}$, and $\mathrm{P}_{\mathrm{ca}}$ are selected with proposed technique. The reason of not selecting $\mathrm{RH}_{\mathrm{ca}}$ and $\mathrm{RH}_{\mathrm{an}}$ in previous studies is that they could not be analysed with developed PEM fuel cell model, due to the assumptions used in the model development. Therefore, the proposed technique can be better applied in practical applications where system physical model cannot be developed accurately.

It should be mentioned that the reason of using multiple sensors instead of voltage only in PEM fuel cell fault diagnosis is that although PEM fuel cell performance decay could be expressed with fuel cell voltage, the PEM fuel cell faults may not be discriminated with good quality, as voltage alone could not provide distinguishable information for various PEM fuel cell faults ${ }^{[28-29]}$. On the other side, since various PEM fuel cell faults or PEM fuel cell faults at different levels could cause variation in different sensors ${ }^{[27]}$, the use of multiple sensors, especially those sensitive to the PEM fuel cell voltage change, could provide better diagnostic results.

\section{Performance of selected sensors in PEM fuel cell system fault diagnosis}

In this section, the proposed sensor selection technique will be applied to a PEM fuel cell system, and the performance of selected sensors in identifying PEM fuel cell fault will be investigated using data-based fault diagnostic techniques. It should be noted that in this study the capability of identifying fuel cell fault is evaluated by determining the different fault severities, to make sure early stage fuel cell fault can be identified accurately. Moreover, the 
capability of evaluating sensor reliability during PEM fuel cell operation will also be illustrated using the proposed technique.

\subsection{Performance of selected sensors in identifying PEM fuel cell fault}

In this section, the performance of selected sensors in PEM fuel cell fault diagnosis will be investigated using data-driven approaches. As high dimensional dataset will be obtained from multiple sensors, KPCA is used herein to reduce the dimension of collected dataset while retaining the useful information; with processed dataset, WPT is applied to extract the wavelet packets and generate the features; finally, SVD is employed to select features which make the most contribution to the state discrimination. Figure 3 depicts the flowchart of the data-driven fault diagnostic framework, more details of these techniques can be found in previous studies ${ }^{[8,15,18]}$.

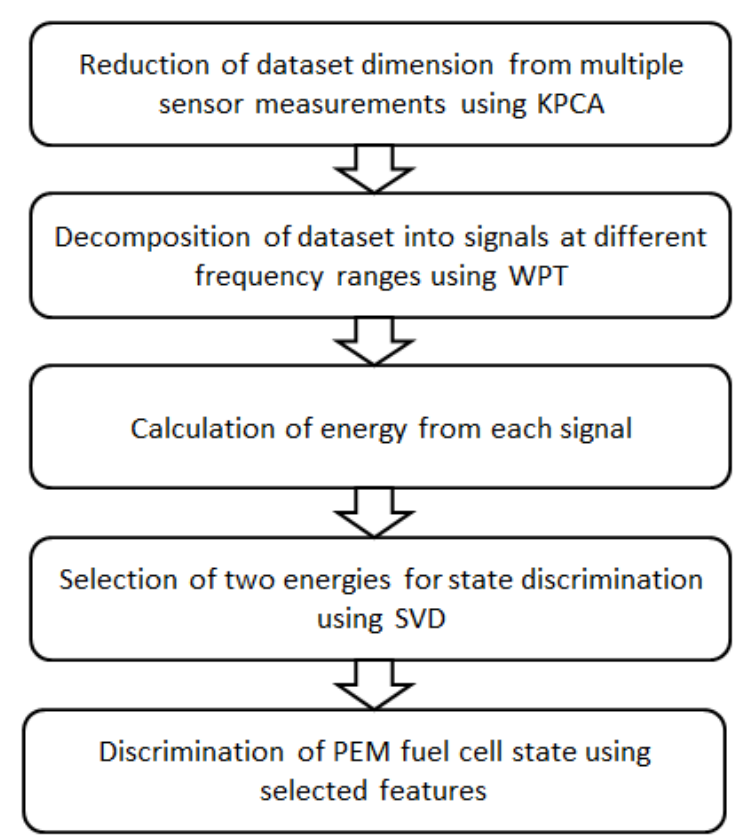

Figure 3 Flowchart of data-driven framework for PEM fuel cell fault diagnosis

To better illustrate the effectiveness of selected sensors in PEM fuel cell fault diagnosis, the diagnostic results using selected sensors is compared to those utilizing all available sensors. Moreover, as the accumulation of liquid water in the test causes the gradual voltage drop, 
PEM fuel cell flooding at different levels are defined, i.e. voltage drop less than 5\% is defined as minor flooding, voltage drop between $5 \%$ and $10 \%$ is defined as medium flooding, and voltage drop larger than $10 \%$ is defined as major flooding. It should be mentioned that in the fault diagnostic analysis, only the test data at the $1^{\text {st }}$ test cycle are used, as from the $2^{\text {nd }}$ cycle, unreliable measurements from some sensors are observed, which will be further described in the next section. Moreover, as the PEM fuel cell temperature is reduced in the test, to make that lower than cathode gas dew point temperature, thus water condensation at cathode is induced, and the voltage drop in the test is caused by PEM fuel cell flooding.

Figures 4 shows the PEM fuel cell fault diagnostic results using all available sensors and selected sensors, respectively. In the analysis, the original dataset is projected to the first two principal directions using KPCA (correspond to $1^{\text {st }}$ and $2^{\text {nd }}$ principal directions in Figure 4), then energies of signals at different frequency ranges are calculated using coefficients from WPT. Among calculated signal energies, SVD is then applied to select two optimal energies for fault diagnosis, which correspond to feature 1 and feature 2 in Figure 4 . Tables 3 and 4 list the confusion matrix of diagnostic results with all available sensors and selected sensors, respectively. It should be noted that the data length of 3 minute is used in the analysis, this is selected since 3 minute flooding would not cause significant voltage drop, thus PEM fuel cell system behaviour during this period can be regarded as the same state. Moreover, this same data length is used in the following section when identifying abnormal sensors.
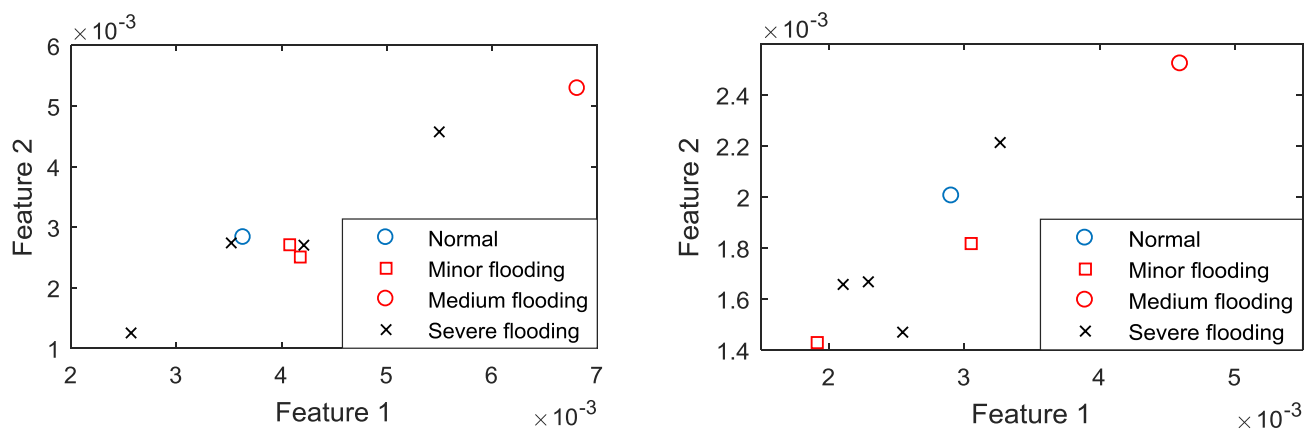
(a) 1 st principal direction with all sensors

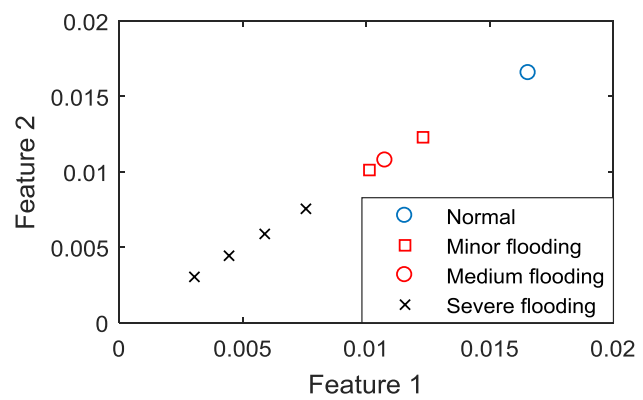

(c) 1 st principal direction with selected sensors (b) 2nd principal direction with all sensors

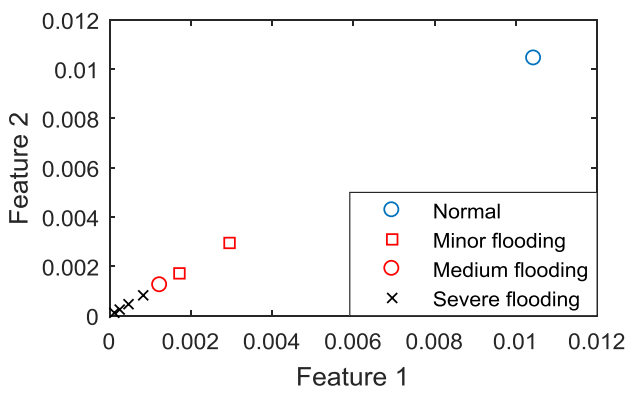

(d) 2nd principal direction with selected sensors

Figure 4 Diagnostic results using all the sensors and selected sensor from proposed technique

Table 3 Confusion matrix with all available sensors

\begin{tabular}{|c|c|c|c|c|c|}
\hline \multirow{2}{*}{\multicolumn{2}{|c|}{}} & \multicolumn{4}{c|}{ Diagnostic results } \\
\cline { 3 - 6 } & & Normal & Minor flooding & Medium & Severe flooding \\
& & & & & flooding \\
Actual & Normal & & & $0 \%$ & $0 \%$ \\
\cline { 3 - 6 } & Minor flooding & $0 \%$ & $75 \%$ & $0 \%$ & $25 \%$ \\
\cline { 2 - 6 } & Medium flooding & $0 \%$ & $0 \%$ & $100 \%$ & $0 \%$ \\
\cline { 2 - 6 } & Severe flooding & $0 \%$ & $12.5 \%$ & $12.5 \%$ & $75 \%$ \\
\hline
\end{tabular}

Table 4 Confusion matrix with selected sensors

\begin{tabular}{|c|c|c|c|c|c|}
\hline \multirow{2}{*}{\multicolumn{2}{|c|}{}} & \multicolumn{4}{|c|}{ Diagnostic results } \\
\cline { 3 - 6 } & & Normal & Minor flooding & Medium & Severe flooding \\
& & & & \\
flooding & \\
\hline \multirow{3}{*}{$\begin{array}{c}\text { Actual } \\
\text { results }\end{array}$} & Normal & $100 \%$ & $0 \%$ & $0 \%$ & $0 \%$ \\
\cline { 2 - 6 } & Minor flooding & $0 \%$ & $75 \%$ & $25 \%$ & $0 \%$ \\
\cline { 2 - 6 } & Medium flooding & $0 \%$ & $0 \%$ & $100 \%$ & $100 \%$ \\
\cline { 2 - 6 } & Severe flooding & $0 \%$ & $0 \%$ & $0 \%$ & $0 \%$ \\
\hline
\end{tabular}


It can be seen that with all available sensors, different PEM fuel cell states cannot be discriminated with good quality, i.e., the normal state would be misclassified as minor flooding, and several minor and severe flooding scenarios could not be distinguished correctly. This confirms that the inclusion of sensors insensitive to the fuel cell performance change would provide negative contributions to the diagnostic performance. On the other side, with selected sensors, different PEM fuel cell states can be classified with good quality, including the normal and flooding states, and flooding scenarios at different levels, as depicted in Figure 4(c) and (d) and Table 4. It should be noted that the discrimination of minor flooding from the normal state can be of great beneficial in practical applications, since the PEM fuel cell performance could be recovered effectively with appropriate mitigations. However, in Figure 4(c) and Table 4, one minor flooding state in the $1^{\text {st }}$ principal direction is misclassified as medium flooding, this is due to the very similar voltage measurements at these two states.

\subsection{Effectiveness of proposed technique in evaluating sensor reliability}

As described in section 2, the proposed sensor selection approach can also be used to evaluate the sensor reliability during the fuel cell system operation. This will be further investigated in this section using test data from the PEM fuel cell system.

In the test, the humidity is obtained using a custom manufactured humidification sensor Tpiece (shown in the top part of Figure 5a), where the gas leaving the humidifiers could be measured by filling the humidifier into the gas tube (shown in the bottom part of Figure 5a). Figure 5(b)-Figure 5(d) depicts the temperature, cathode relative humidity, and corresponding fuel cell voltage measurements in the test. It can be seen that cathode relative humidity has sudden drop just after about 5000s ( $2^{\text {nd }}$ cycle of temperature change), while fuel cell voltage does not show variation at this time (a slight voltage drop is due to the fact that 
characterization test performed before 5000s leads to voltage jump within a short period, then reduce to normal value), indicating no performance decay of PEM fuel cell system. Therefore, it can be confirmed that without PEM fuel cell performance decay, the variation in cathode relative humidity is due to the abnormal humidification sensor at cathode outlet. With further investigation, it is found that the abnormal measurements from cathode humidification sensor is due to the accumulation of liquid water on the humidification sensor surface, which does not represent the actual cathode humidity.

It should be mentioned that the noisy cathode relative humidity measurements are obtained, as shown in Figure 5(c), where relative humidity higher than $100 \%$ is observed, this is due to the measurement noise. However, as the humidification sensor malfunction causes significant variation in the cathode humidity measurement, i.e., the variation due to humidification sensor malfunction is much larger than that from noise, thus the use of raw cathode relative humidity data in the analysis will not affect the diagnostic results, and the variation due to sensor malfunction can still be identified and analyzed. However, in cases where only minor measurement variation is caused by sensor malfunction, pre-processing techniques including filtering and smoothing methods should be applied to remove/reduce the noise influence.

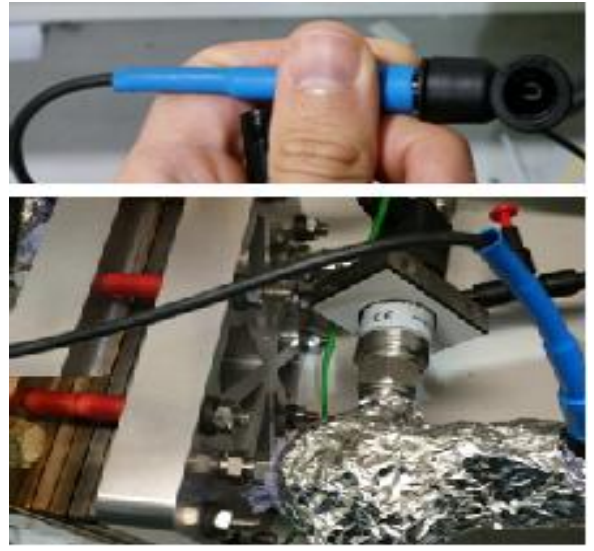

(a) Humidification sensor

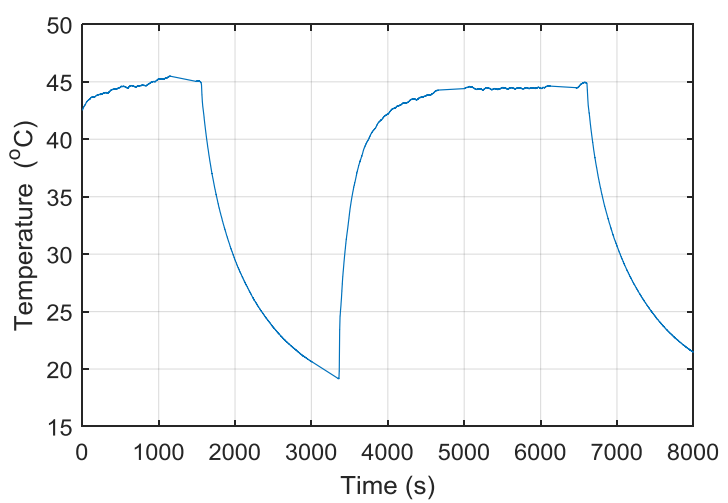

(b) Fuel cell temperature 


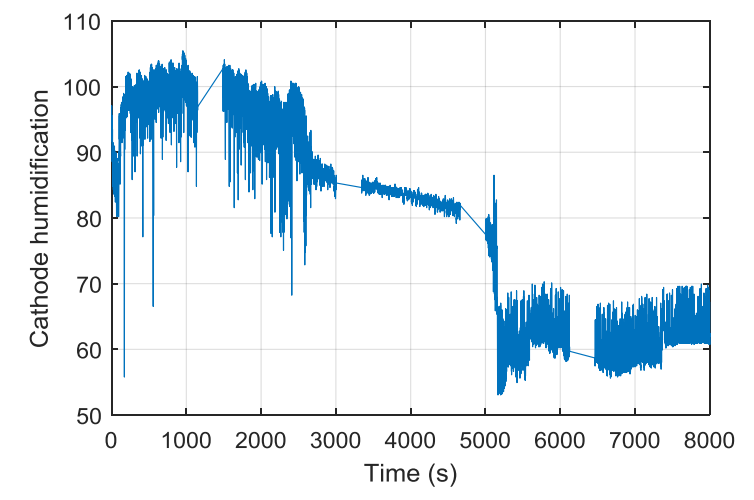

(c) Cathode relative humidity

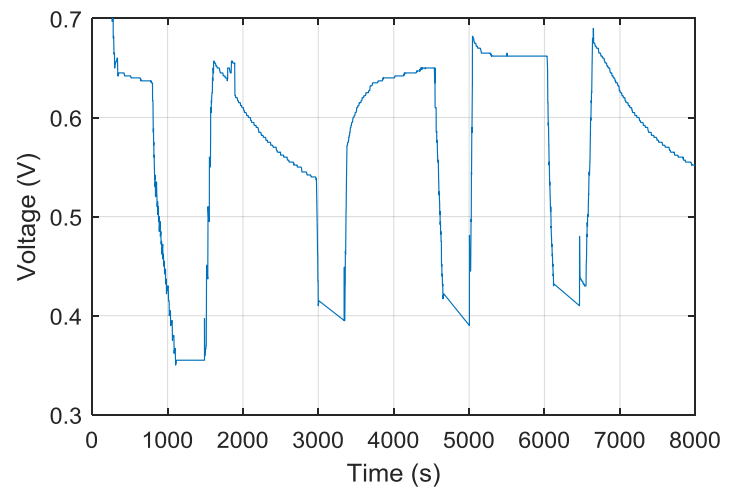

(d) Fuel cell voltage

Figure 5 Humidification sensor T-piece detail and its measurement during the test

The effect of abnormal sensor measurements on PEM fuel cell fault diagnostic results should be investigated, thus the necessity of identifying abnormal sensors in PEM fuel cell fault diagnosis could be further clarified. In this study, the data-driven fault diagnostic approaches illustrated in Figure 3 are applied to the selected sensors (including unreliable cathode humidification sensor), and the diagnostic results at two principal directions are depicted in Figure 6. Table 5 lists the confusion matrix of diagnostic results with abnormal cathode humidity sensor. Compared with diagnostic results in Figure 4(c) and (d), it can be observed that the diagnostic performance is greatly affected by inclusion of abnormal sensor measurements, where only normal PEM fuel cell state can be discriminated from the fuel cell flooding, while flooding scenarios at different levels cannot be classified with good quality, which hinders the use of efficient mitigation strategies for recovering PEM fuel cell performance at its early fault stage. 


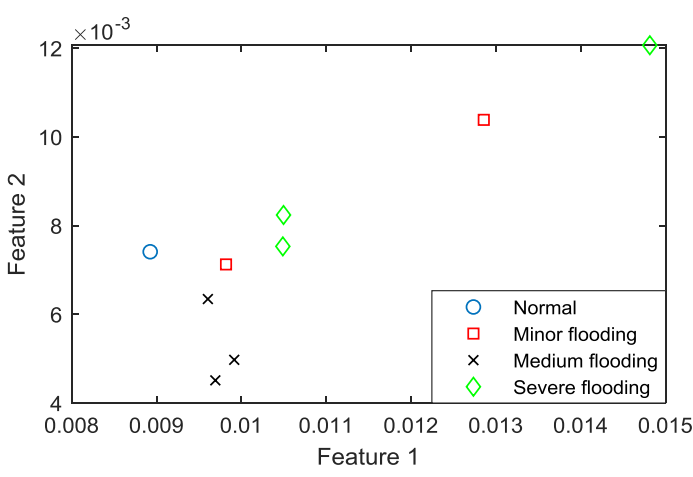

(a) 1st principal direction

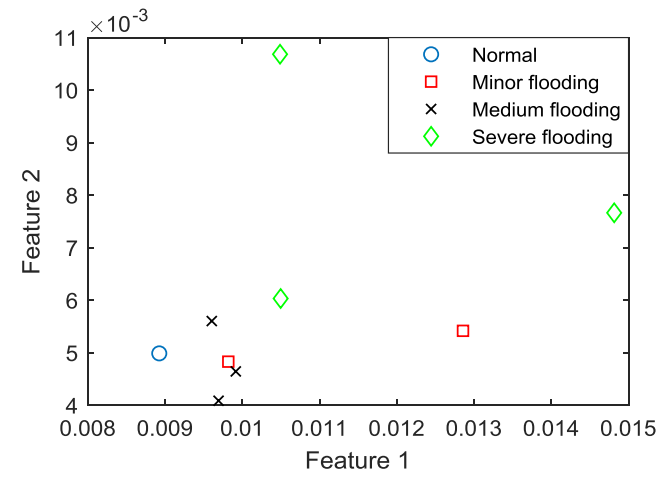

(b) 2nd principal direction

Figure 6 Diagnostic results using selected sensor including abnormal cathode humidity

Table 5 Confusion matrix with abnormal cathode humidification sensor

\begin{tabular}{|c|c|c|c|c|c|}
\hline \multirow{2}{*}{\multicolumn{2}{|c|}{}} & \multicolumn{3}{|c|}{ Diagnostic results } \\
\cline { 3 - 6 } & & Normal & Minor flooding & Medium & Severe flooding \\
& & & & \\
flooding & & $0 \%$ \\
\hline \multirow{3}{*}{$\begin{array}{l}\text { Actual } \\
\text { results }\end{array}$} & Normal & $100 \%$ & $0 \%$ & $0 \%$ & $0 \%$ \\
\cline { 2 - 6 } & Minor flooding & $25 \%$ & $50 \%$ & $25 \%$ & $50 \%$ \\
\cline { 2 - 6 } & Medium flooding & $33 \%$ & $0 \%$ & $67 \%$ & $50 \%$ \\
\hline
\end{tabular}

As described in section 2, the consistency of various sensors calculated using Eq.(1) can be used for evaluation of sensor reliability, results are depicted in Figure 7. It can be found that extremely high consistency value from cathode relative humidity measurements is obtained, while consistency of other sensors are located at similar level. Considering the fact that PEM fuel cell voltage does not decay during this period, the consistency variation in cathode relative humidity is due to the loss of cathode humidification sensor reliability. 


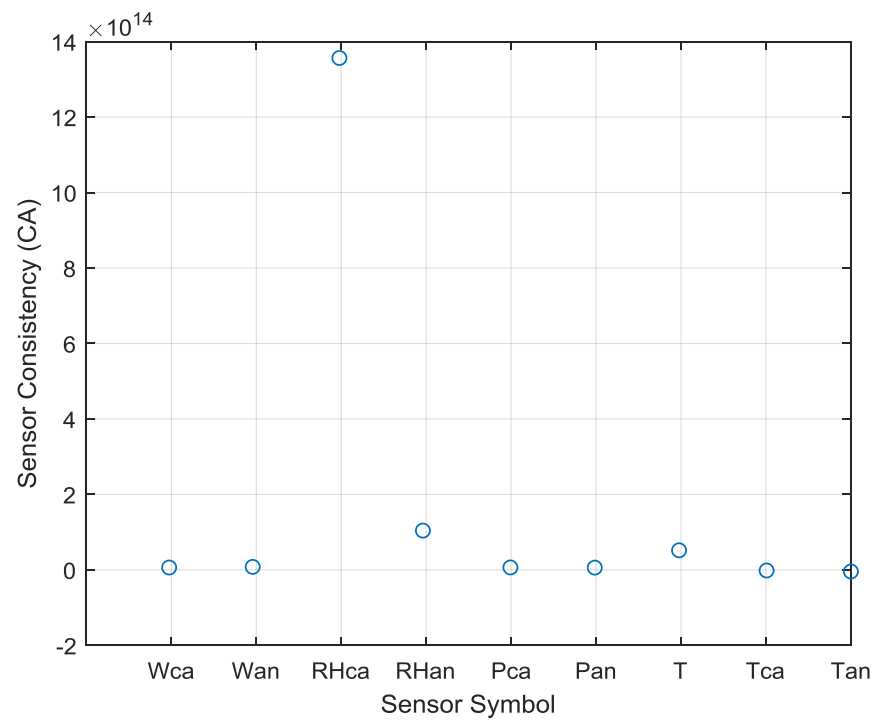

Figure 7 Sensor capability for identification of abnormal sensors

From results in Figure 7, the measurements of abnormal cathode humidification sensor should be excluded from fault diagnosis, i.e., only four sensors ( $\mathrm{T}, \mathrm{T}_{\mathrm{ca}}, \mathrm{P}_{\mathrm{ca}}$, and $\mathrm{RH}_{\mathrm{an}}$ ) should be selected for PEM fuel cell fault diagnosis. Figure 8 depicts the diagnostic results using the four selected sensors, where data-driven approaches shown in Figure 3 are applied. The confusion matrix of diagnostic results is listed in Table 6. It can be seen that without the inclusion of abnormal cathode humidification sensor, only one minor flooding scenario is misclassified as normal state, while other different PEM fuel cell states, including normal and flooding scenarios, and flooding at different levels, can be discriminated accurately.

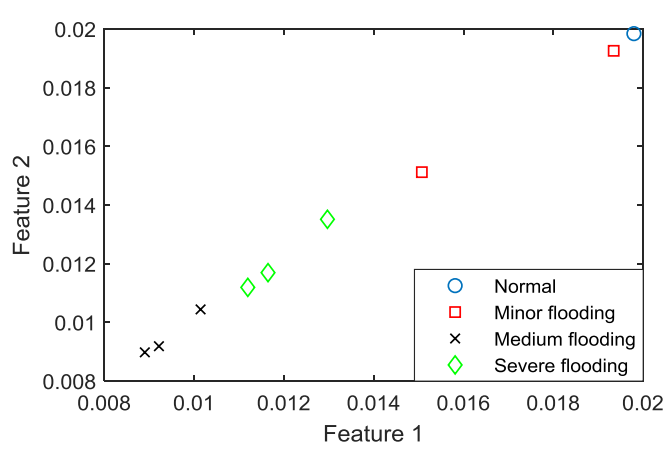

(a) 1st principal direction

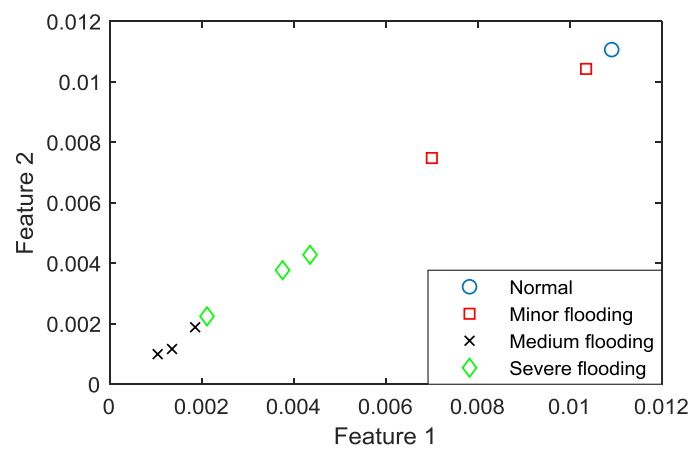

(b) 2nd principal direction 
Figure 8 Diagnostic results using selected sensor without abnormal cathode humidity

Table 6 Confusion matrix without abnormal cathode humidification sensor

\begin{tabular}{|c|c|c|c|c|c|}
\hline \multirow{2}{*}{\multicolumn{2}{|c|}{}} & \multicolumn{3}{|c|}{ Diagnostic results } \\
\cline { 3 - 6 } & & Normal & Minor & Medium & Severe \\
& & & flooding & flooding & flooding \\
\hline \multirow{3}{*}{ Actual } & Normal & $100 \%$ & $0 \%$ & $0 \%$ & $0 \%$ \\
\cline { 2 - 6 } & Minor flooding & $50 \%$ & $50 \%$ & $0 \%$ & $0 \%$ \\
\cline { 2 - 6 } & Medium flooding & $0 \%$ & $0 \%$ & $100 \%$ & $100 \%$ \\
\cline { 2 - 6 } & Severe flooding & $0 \%$ & $0 \%$ & $0 \%$ & $0 \%$ \\
\hline
\end{tabular}

\section{Conclusion}

In the study, a novel sensor selection technique is proposed without the use of polymer electrolyte membrane fuel cell physical model, which is more beneficial in practical systems, where development of accurate fuel cell physical model is extremely difficult or even impossible. Moreover, with the proposed technique, the reliability of sensors during the system operation can be evaluated. With identification and removal of abnormal sensors from fault diagnosis, reliable diagnostic performance could be provided.

The test data from a polymer electrolyte membrane fuel cell system is used to investigate the performance of proposed technique, where fuel cell flooding scenarios at different levels are observed. Compared with the sensor selection results from previous studies, the proposed technique can provide consistent sensor selection results, and sensors cannot be analysed in previous algorithms due to the physical model limitations can also be investigated with proposed technique. With the selected sensors, reliable fault diagnostic performance can be provided using data-driven fault diagnostic approaches, where different levels of fuel cell flooding can be discriminated with good quality. 
Furthermore, the effectiveness of proposed technique in evaluating sensor reliability is studied using the polymer electrolyte membrane fuel cell test data, where abnormal cathode relative humidity measurements are observed in the test. The effect of abnormal sensor measurements on polymer electrolyte membrane fuel cell fault diagnostic performance is firstly investigated. Results demonstrate that the use of abnormal sensors can greatly affect the diagnostic performance. With proposed technique, the abnormal cathode humidification sensor can be identified, and by removing the abnormal sensor measurements from the analysis, fuel cell flooding, including the minor flooding scenario, can be discriminated accurately.

Therefore, with proposed technique, various failures in practical polymer electrolyte membrane fuel cell systems, including fuel cell faults (flooding in the study), and faults of other ancillary systems (sensors in the study), could be identified with good quality. From the results, faults at polymer electrolyte membrane fuel cell could be identified at their early stage, such that mitigation strategies could be taken to recover the system performance and also extend the system useful lifetime, moreover, false alarms due to abnormal sensors could also be detected with proposed method, which could reduce the unnecessary cost of system stop and maintenance.

It should be mentioned that the proposed sensor selection technique can also be used in other systems for identifying system and sensor faults. As system physical model is not required, the sensor selection and reliability evaluation can be performed using the sensor measurements continuously collected from the system, which will not interrupt the system operation. Moreover, as fewer sensors are selected for the fault isolation, the fault diagnosis can be performed more efficiently, which can be better used for the system on-line monitoring. In the future, the effectiveness of proposed method on detection and isolation of PEM fuel cell faults, including hydrophobicity property change of gas diffusion medium, and 
surface energy loss on the bipolar plates, will be further investigated, since these faults can lead to significant PEM fuel cell performance loss.

\section{Acknowledgment}

This work is supported by grants 'National Natural Science Foundation of China (NSFC) [grant number 51975549]', 'Anhui Provincial Natural Science Foundation [grant number 1908085ME161]', and 'EP/K02101X/1 for Loughborough University, Department of Aeronautical and Automotive Engineering from the UK Engineering and Physical Sciences Research Council (EPSRC)'.

\section{Reference}

[1] Petrone, R., Zheng, Z., Hissel, D., Pera, M.C., Pianese, C., Sorrentino, M., Becherif, M., Yousfi-Steiner, N. A review on model-based diagnosis methodologies for PEMFCs, International Journal of Hydrogen Energy, 38(2013) 7077-7091.

[2] Forrai, A., Funato, H., Yanagita, Y., Kato, Y. Fuel-cell parameter estimation and diagnostics, IEEE Transactions on Energy Conversion, 20(2005) 668-675.

[3] Fouquet, N., Doulet, C., Nouillant, C., Tanguy, G.D., Bouamama, B.O. Model based PEM fuel cell state-of-health monitoring via ac impedance measurements, Journal of Power Sources, 159(2006) 905-913.

[4] Ingimundarson, A., Stefanopoulou, A.G., McKay, D.A. Model-based detection of hydrogen leaks in a fuel cell stack, IEEE Transactions on Control Systems Technology, 16(2008) 1004-1012.

[5] Rubio, M.A., Urquia, A., Dormido, S. Diagnosis of performance degradation phenomenon in PEM fuel cells, International Journal of Hydrogen Energy, 35(2010) 2586-2590. 
[6] Kamal, M.M., Yu, D. Model-based fault detection for proton exchange membrane fuel cell systems, International Journal of Engineering, Science and Technology, 3(2011) 115.

[7] Mohammadi, A., Djerdir, A., Bouquain, D., Bouriot, B., Khaburi, D. Fault sensitive modeling and diagnosis of PEM fuel cell for automotive applications, Transportation Electrification Conference and Expo (ITEC), Detroit, USA, 2013.

[8] Zheng, Z., Petrone, R., Pera, M.C., Hissel, D., Becherif, M., Pianese, C., Steiner, N.Y., Sorrentino, M. A review on non-model based diagnosis methodologies for PEM fuel cell stacks and systems, International Journal of Hydrogen Energy, 38(2013) 8914-8926.

[9] Andujar J.M., Segura F., Isorna F., Calderon A.J. Comprehensive diagnosis methodology for faults detection and identification, and performance improvement of air-cooled polymer electrolyte fuel cells. Renewable and Sustainable Energy Reviews, 88(2018) 193-207.

[10] Lin R.H., Xi X.N., Wang P.N., Wu B.D., Tian S.M. Review on hydrogen fuel cell condition monitoring and prediction methods. International Journal of Hydrogen Energy, 44(2019) 5488-5498.

[11] Steiner, N.Y., Hissel, D., Mocoteguy, P., Candusso, D. Non intrusive diagnosis of polymer electrolyte fuel cells by wavelet packet transform, International Journal of Hydrogen Energy, 36(2011) 740-746.

[12] Zhongliang, L., Outbib, R., Giurgea, S., Hissel, D., Li, Y. Fault detection and isolation for polymer electrolyte membrane fuel cell systems by analysing cell voltage generated space, Applied Energy, 148(2015) 260-272.

[13] Kim, J.G., Mukherjee, S., Bates, A., Zickel, B., Park, S., Son, B.R., Choi, J.S., Kwon, O., Lee, D.H., Chung, H.Y. Autocorrelation standard deviation and root mean square 
frequency analysis of polymer electrolyte membrane fuel cell to monitor for hydrogen and air undersupply, Journal of Power Sources, 300(2015) 164-174.

[14] Liu, J., Li, Q., Chen, W., Yan, Y., Wang, X. A fast fault diagnosis methode of the PEMFC system based on extreme learning machine and Dempster-Shafer evidence theory, IEEE Transactions on Transportation Electrification, 5(2019) 271-284.

[15] Placca, L., Kouta, R., Candusso, D., Blachot, J.F., Charon, W. Analysis of PEM fuel cell experimental data using principle component analysis and multi linear regression, International Journal of Hydrogen Energy, 35(2010) 4582-4591.

[16] Pahon, E., Steiner, N.Y., Jemei, S., Hissel, D., Mocoteguy, P. A signal-based method for fast PEMFC diagnosis, Applied Energy, 165(2016) 748-758.

[17] Liu J., Li Q., Chen W., Cao T. A discrete hidden Markov model fault diagnosis strategy based on K-means clustering dedicated to PEM fuel cell systems of tramways. International Journal of Hydrogen Energy, 43(2018) 12428-12441.

[18] Mao, L., Jackson, L.M., Dunnett, S.J. Fault diagnosis of practical polymer electrolyte membrane (PEM) fuel cell system with data-driven approaches, Fuel Cells, 17(2017) 247-258.

[19] Vural, Y., Ingham, D.B., Pourkashanian, M. Performance prediction of a proton exchange membrane fuel cell using the ANFIS model, International Journal of Hydrogen Energy 34(2009) 9181-9187.

[20] Becker, S., Karri, V. Predictive models for PEM-electrolyzer performance using adaptive neuro-fuzzy inference systems, International Journal of Hydrogen Energy 35(2010) 99639972.

[21] Silva, R.E., Gouriveau, R., Jemei, S., Hissel, D., Boulon, L., Agbossou, K., Steiner, N.Y. Proton exchange membrane fuel cell degradation prediction based on adaptive neurofuzzy inference systems, International Journal of Hydrogen Energy 39(2014) 1-17. 
[22] Liu J., Li Q., Chen W., Yan Y., Qiu Y., Cao T. Remaining useful life prediction of PEMFC based on long short-term memory recurrent neural networks. International Journal of Hydrogen Energy, 44(2019) 5470-5480.

[23] Ma R., Yang T., Breazab E., Li Z., Brioise P., Cao F. Data-driven proton exchange membrane fuel cell degradation prediction through deep learning method. Applied Energy, 231(2018) 102-115.

[24] Kishor N., Mohanty S.R. Fuzzy modeling of fuel cell based on mutual information between variables. International Journal of Hydrogen Energy, 35(2010) 3620-3631.

[25] Li Q., Yang H., Han Y., Li M., Chen W. A state machine strategy based on droop control for an energy management system of PEMFC-battery-supercapacitor hybrid tramway. International Journal of Hydrogen Energy, 41(2-16) 16148-16159.

[26] Li Q., Wang T., Dai C., Chen W., Ma L. Power management strategy based on adaptive droop control for a fuel cell-battery-supercapacitor hybrid tramway. IEEE Transactions on Vehicle Technology, 67(2018) 5658-5670.

[27] Wu, J., Yuan X.Z., Martin, J.J., Wang, H., Zhang, J., Shen, J., Wu, S., Merida, W. A review of PEM fuel cell durability: degradation mechanisms and mitigation strategies, Journal of Power Sources. 184(2008) 104-119.

[28] Riascos, L.A.M., Simoes, M.G., Miyagi, P.E. On-line fault diagnostic system for proton exchange membrane fuel cells. Journal of Power Sources, 175(2008) 419-429.

[29] Davies, B., Jackson, L., Dunnett, S. Expert diagnosis of polymer electrolyte fuel cells. International Journal of Hydrogen Energy, 42(2017) 11724-11734.

[30] Mawardi, A., Pitchumani, R, Effects of parameter uncertainty on the performance variability of proton exchange membrane (PEM) fuel cells, Journal of Power Sources 160(2006) 232-245. 
[31] Placca, L., Kouta, R., Blachot, J., Charon, W, Effects of temperature uncertainty on the performance of a degrading PEM fuel cell model, Journal of Power Sources 194(2009) 313-327.

[32] Correa, G., Borello, F., Santarelli, M, Sensitivity analysis of temperature uncertainty in an aircraft PEM fuel cell, International Journal of Hydrogen Energy 36(2011) 14745-14758.

[33] Noorkami, M., Robinson, J., Meyer, Q., Obeisun, O., Fraga, E., Reisch, T, Effects of temperature uncertainty on polymer electrolyte fuel cell performance, International Journal of Hydrogen Energy, 39(2014) 1439-1448.

[34] Correa, G., Santarelli, M., Borello, F, Sensitivity analysis of stack power uncertainty in a PEMFC-based powertrain for aircraft application, International Journal of Hydrogen Energy, 40(2015) 10354-10365.

[35] Mao, L., Jackson, L.J. Selection of optimal sensors for predicting performance polymer electrolyte membrane fuel cell. Journal of Power Sources, 328(2016) 151-160.

[36] Mao, L., Jackson, L.J., Davies, B., Jackson, T., Dunnett, S.J. Effectiveness of a novel sensor selection algorithm in PEM fuel cell on-line diagnosis, IEEE Transactions on Industrial Electronics.

[37] Mao, L., Davies, B., Jackson, L.J. Application of sensor selection approach in polymer electrolyte membrane fuel cell prognostics and health management, Energies, 10(2017) 1511.

[38] Li S., Aitouche A., Wang H., Christov N. Sensor fault estimation of PEM fuel cells using Takagi Sugeno fuzzy model. International Journal of Hydrogen Energy, doi.org/10.1016/j.ijhydene.2019.01.100

[39] Mao, L., Jackson, L., Davies, B. Investigation of PEMFC fault diagnosis with consideration of sensor reliability. International Journal of Hydrogen Energy, 43(2018) 16941-16948. 